Tropical Journal of Pharmaceutical Research May 2020; 19 (5): 995-999

ISSN: $1596-5996$ (print); 1596-9827 (electronic) (C) Pharmacotherapy Group, Faculty of Pharmacy, University of Benin, Benin City, 300001 Nigeria.

\title{
Albiflorin attenuates inflammation and apoptosis by upregulating AMPK-mediated expression of CDX2 in a mouse model of ulcerative colitis
}

\author{
Xiaoping $\mathrm{Xu}^{1}$, Haiyan Liu², Yuan Pan¹, Zhaohui Liu1, Dong Chen, Lei Zhang1, \\ Hong Yuang ${ }^{3 *}$ \\ ${ }^{1}$ Department of Anorectal Surgery, Yuhang District First People's Hospital, Hangzhou City, Zhejiang Province 311100 , \\ ${ }^{2}$ Department of Dermatology, Hunan Academy of Traditional Chinese Medicine Affiliated Hospital, Changsha City, Hunan \\ Province 410006, ${ }^{3}$ Department of Cardiology, Yuhang District First People's Hospital, Hangzhou City, Zhejiang Province \\ 311100, China
}

*For correspondence: Email: HongYuangkl@163.com; Tel: +86-571-89369151

Sent for review: 10 February 2020

Revised accepted: 28 April 2020

\begin{abstract}
Purpose: To investigate the mechanism underlying the ameliorative effect of albiflorin (AF) on ulcerative colitis (UC) in dextran sulphate sodium (DSS)-induced mice model.

Method: Female C57BL/6 mice were administered DSS to establish a mice model of UC. After one week, the mice received $A F$, and the body weight and length of colon were measured. The histopathological features of colon tissues treated with hematoxylin-eosin $(H \& E)$ stain were examined by microscopy. Expression of inflammatory cytokines and apoptosis-related proteins were determined using enzyme-linked immunosorbent assay (ELISA) and western blotting.

Results: The relative abundance of goblet cells and crypts of mice were significantly reduced in DSSinduced UC mice model; furthermore, focal ulcers and mucosal damage were apparent. Moreover, treatment with DSS decreased body weight and colon length, downregulated Bcl-2 and AMPK pathwayrelated proteins, increased inflammatory cytokines levels, and upregulated Bax and cleaved caspase-3. In contrast, treatment with AF completely ameliorated DSS-induced effects.

Conclusion: AF treatment attenuated DSS-induced inflammation response and apoptosis via AMPK pathway and modulation of CDX2 expression in UC mice model.
\end{abstract}

Keyword: Albiflorin, Ulcerative colitis, AMPK, CDX2, Apoptosis

\begin{abstract}
This is an Open Access article that uses a fund-ing model which does not charge readers or their institutions for access and distributed under the terms of the Creative Commons Attribution License (http://creativecommons.org/licenses/by/4.0) and the Budapest Open Access Initiative (http://www.budapestopenaccessinitiative.org/read), which permit unrestricted use, distribution, and reproduction in any medium, provided the original work is properly credited.

Tropical Journal of Pharmaceutical Research is indexed by Science Citation Index (SciSearch), Scopus, International Pharmaceutical Abstract, Chemical Abstracts, Embase, Index Copernicus, EBSCO, African Index Medicus, JournalSeek, Journal Citation Reports/Science Edition, Directory of Open Access Journals (DOAJ), African Journal Online, Bioline International, Open-J-Gate and Pharmacy Abstracts
\end{abstract}

\section{INTRODUCTION}

Ulcerative colitis (UC) is a chronic non-specific inflammatory disease characterized by inflammation and ulcers of rectum and colonic mucosa [1]. It is characterized by recurrent abdominal pain, diarrhea, and mucopurulent bloody stool [1]. The age of UC onset has been steadily decreased and its incidence has recently increased. Though previous studies have focused on infection, immunity, genetics, and environmental factors, the etiology and pathogenesis of UC have not been fully defined [2]. It is found that immune dysfunction is a main 
cause of UC via the involvement of intestinal hyperfunction [2]. Neutrophils, mast cells, macrophages, B lymphocytes cells, T cells and natural killer cells are involved in the pathogenesis of UCby releasing antibodies, cytokines and inflammatory mediators to destroy and damage tissues[1,3]. Albiflorin $(A F)$, also known as 9-((benzoyl) methyl) -1- (B-Dglucopyranosyloxy) - 4 -hydroxy-6-methyl-7oxotricyclononane-8-ketone, is a monoterpene glycoside component extracted from the root of Paeonia tacti lora Pall [4]. It has been recently found that AF has pharmacodynamic characteristics that are unique from paeoniflorin, another compound isolated from the same plant [4]. Albiflorin has also been shown to have potential benefits in the treatment of blood pressure, depression, and diabetes [4].

A previous study reported that $A F$ reduced weight through modulation of adenosine 54monophosphate activated protein kinase (AMPK) and phosphatidylinositol 3-kinase (PI3K)/protein kinase $B$ (AKT) pathways [5]. However, there are few researches considering the effects of $A F$ on UC. Although AF could alleviate UC by regulating signaling through toll-like receptor 4 (TLR4), the detailed molecular mechanisms remain unclear [6]. Here, DSS was used to establish UC mice model and to study the mechanisms underlying the functional role of AF on UC, providing a solid foundation for the use of AF as a new treatment method for UC.

\section{EXPERIMENTAL}

\section{Animal model}

Female C57BL/6 mice ( $\mathrm{n}=18,8$ weeks, 18 - 24 g) were purchased from Guangdong Medical Laboratory Animal Center (Foshan, China) and randomly grouped: sham group, DSS group, and DSS + AF group ( $n=6$ per group). Briefly, DSS mice were received $10 \mathrm{~mL}$ distilled water containing 4 \% DSS (MW: 36000 - 50000 Da, Seebio, Shanghai, China). DSS + AF mice were given $10 \mathrm{~mL}$ distilled water with $4 \%$ DSS, and then intraperitoneally injected with AF $(1.0 \mathrm{~g} / \mathrm{kg}$ body weight, Wako, Osaka, Japan). Sham mice were given $10 \mathrm{~mL}$ distilled water. These administrations were last for 7days, and the body weight was measured daily. Subsequently, all mice were sacrificed and colons were removed for observation and measurements. All animal experiments were approved by the institutional ethics committee (protocol no. ZSLL-2016-101) and performed in accordance with the guidelines of the National Institutes of Health Guide for the Care and Use of Laboratory Animals [7].

\section{Hematoxylin eosin (H \& E) staining}

Colon tissues from DSS-induced UC mice model were embedded in paraffin, sectioned into histology slides, and washed in ethanol, and finally stained with $H$ \& $E$. The slides were then dehydrated in ethanol and photographed under a light microscope (Zhaoyi, Shanghai, China).

\section{Enzyme-linked immunosorbent assay (ELISA)}

Colon tissues were washed with PBS, and then homogenized, and the supernatant was kept for subsequent steps. The concentrations of tumor necrosis factor- $\alpha$ (TNF- $\alpha$ ), interferon- $\gamma$ (IFN- $\gamma$ ), and interleukin 6 (IL-6) were measured using ELISA Kits (Thermo Fisher Scientific, Waltham, USA).

\section{Western blotting}

Total protein from the colon tissues was isolated, quantified on the basis of molecular weight using SDS-PAGE, and then transferred to nitrocellulose membranes (Sigma-Aldrich, St. Louis, USA). Then, the membranes were blocked with $5 \%$ milk in PBS for $2 \mathrm{~h}$ and then incubated with primary antibodies (Abcam, Cambridge, USA). against B-cell lymphoma-2 (Bcl-2, ab196495), Bcl-2-associated X (Bax, ab182374), cleaved caspase-3 (ab2302), $\beta$-actin (ab8227), AMPK (ab32047), p-AMPK (ab131357), caudaltype homeobox transcription factor-2 (CDX2, ab76541), zonula occludens 1 (ZO-1, ab96587) and villin (ab130751, all 1:1000) at $4{ }^{\circ} \mathrm{C}$ for $16 \mathrm{~h}$. The primary antibodies. The membranes were incubated with secondary antibody (anti-rabbit IgG; ab6721, 1:2,000) for $2 \mathrm{~h}$ at room temperature. The immunoblots were visualized and analyzed.

\section{Statistical analysis}

Data presented as mean \pm standard deviation (SD) obtained from replicate experiments and analyzed by SPSS 21.0 software (SPSS Inc, Chicago, USA). Analysis of variance (ANOVA) and Student's $t$-test were used to assess significant difference. $P<0.05$ was considered statistically significant.

\section{RESULTS}

\section{Treatment of AF alleviated DSS-induced damage in mice}

To study the functional effect of AF on UC mice, DSS was used to establish the mice model. The body weight of DSS mice was lower than that of Sham mice $(p<0.01)$. However, AF treatment 
led to increased body weight when compared to DSS group (Figure 1A; $p<0.05$ ). Figure $1 \mathrm{~B}$ showed that the length of the colon was shorter in DSS-treated mice than Sham mice, however, AF treatment reversed it $(p<0.01)$.

As expected, H\&E staining showed that the colon tissue structure of Sham mice was regular, and the goblet cells and crypts appeared healthy (Figure $1 \mathrm{C}$ ). In DSS group, the goblet cells and crypts of mice were significantly reduced, and focal ulcers and mucosal damage were obvious. However, co-treatment of DSS + AF led to a recovery in the number of goblet cells and crypts and caused a reduction in the degree of focal ulcers and mucosal damage compared to DSS group (Figure 1C). These results revealed that AF alleviated DSS-induced gastrointestinal injury in mice.
A

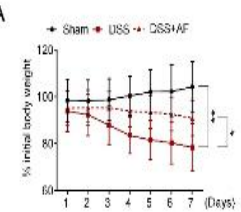

C

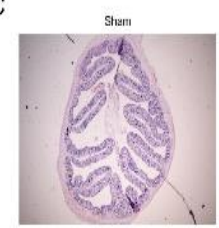

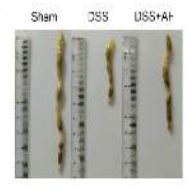
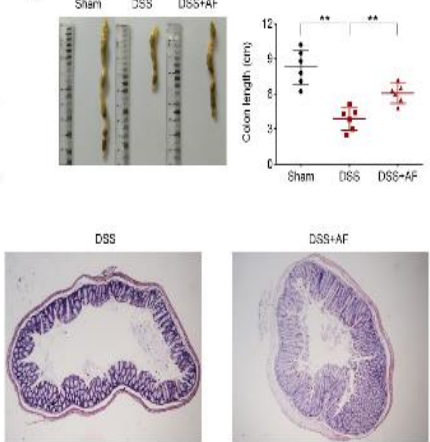

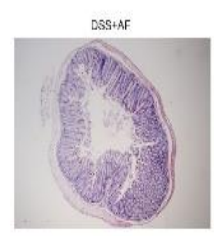

Figure 1: Effect of $A F$ treatment on DSS-induced injury in UC mice model. (A) Treatment of AF alleviated the reduction of the body weights in DSStreated mice. (B) Treatment of AF alleviated the reduction of colon length in DSS-treated mice. (C)Treatment of AF restored the number of goblet cells and crypts, reduced the degree of local ulcers and mucosal damage. ${ }^{*} P<0.05$ and ${ }^{* *} p<0.01$

\section{Effect of AF treatment reduced inflammatory cytokine expression and inhibited apoptosis}

In order to further investigate the mechanism, the levels of inflammatory cytokines and apoptosisrelated proteins were examined in UC mice. Figures $2 \mathrm{~A}-\mathrm{C}$ showed that the concentrations of TNF- $\alpha$, IFN- $y$, and IL- 6 were increased in the DSS group when compared with the Sham group, but the increased concentrations of inflammatory cytokine was attenuated by $A F$ treatment $(p<0.01)$, indicating that AF alleviated inflammation in DSS-induced UC mice model. Moreover, western blotting showed that, in DSS group, Bcl-2 expression was lower, but the protein expression of Bax and cleaved caspase-3 were higher than that in Sham group $(p<0.01$, Figure 3 ). In contrast, these expression levels were reversed by AF treatment $(p<0.01$, Figure 3 ). These data suggest that AF might inhibit cell apoptosis in DSS-induced UC mice model.
A

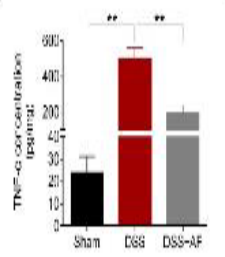

B

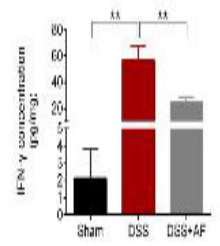

C

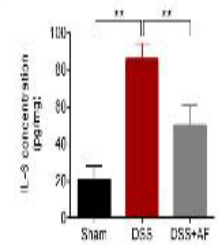

Figure 2: Effect of AF treatment on the levels of inflammatory cytokines, (A) IFN- $y$, (B) IL-6 and (C) TNF- $\alpha$ in the colon of DSS-induced UC mice model. ** $P<0.01$
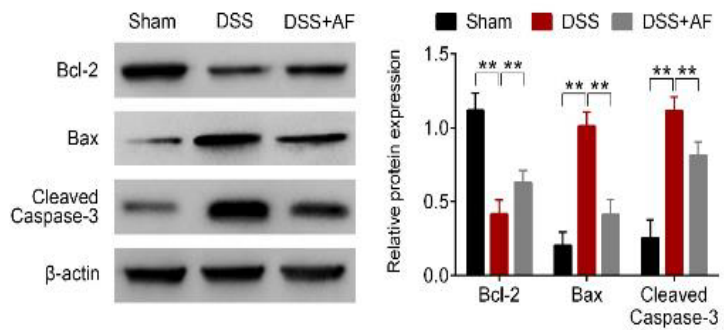

Figure 3: Treatment with $A F$ inhibited apoptosis of enterocytes in DSS-induced UC mice model. Expression of apoptosis-related proteins was analyzed by western blotting; ${ }^{* *} p<0.01$

Treatment with AF alleviated DSS-induced injury by activating AMPK pathway

To study the specific pathways important in the action of AF in the DSS-induced mouse model of UC, western blotting was used to analyze pathway-related protein expressions. The results found that p-AMPK/AMPK, CDX2, ZO-1, and villin levels were downregulated in DSS-treated mice. However, the expression of these proteins was recovered by AF treatment.
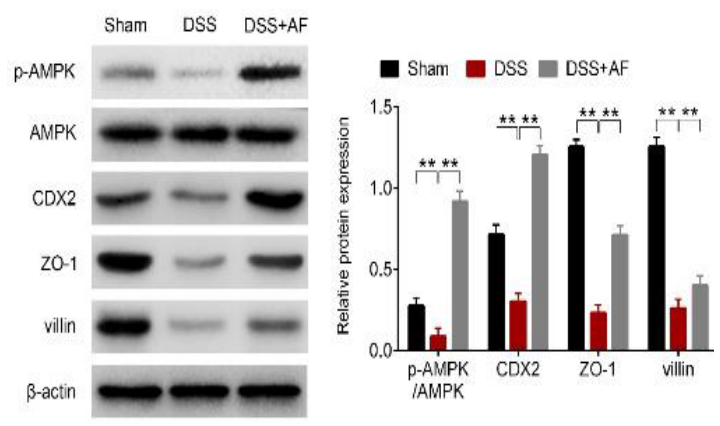

Figure 4: Treatment with AF activated AMPK pathway in DSS-induced UC mice model. The protein expression was analyzed by western blotting; ${ }^{* *} p<$ 0.01 


\section{DISCUSSION}

Ulcerative colitis, a common disease of digestive system, is recurrent and difficult for cure [1]. The World Health Organization (WHO) has listed UC as a modern refractory disease [1]. At present, the etiology of UC has not been fully elucidated, but evidences have suggested that the occurrence of UC is caused by a combination of diverse factors [2]. Particularly, dysregulated immune activity plays a primary role [2]

Common treatments for UC are intended to regulate immune function and reduce inflammatory responses [8]. Indeed, many cytokines play important roles in regulating the occurrence of UC. For example, IFN- $\gamma$, TNF- $\alpha$, $\mathrm{IL}-1 \beta$, and IL-12 activate $\mathrm{T}$ cells or $\mathrm{B}$ cells and induce the production of other inflammatory factors, leading to the accumulation of neutrophils or macrophages at the site of inflammatory area, finally resulting in the damage to intestinal mucosa $[9,10]$. In particular, TNF-a and IL-6 are thought to be main inflammatory factors that mediate intestinal mucosal injury in UC.

Dextran sulphate sodium (DSS) is a sulfated polysaccharide synthesized from sucrose, which has anti-hemostatic and anti-coagulant effects [11].

Treatment with DSS induces animal models of UC and have been widely used in mechanistic and therapeutic studies. In line with those prior studies, this work used a solution of $4 \%$ DSS to establish UC mice model. This study found that DSS treatment increased the concentrations of IFN- $\gamma$, TNF- $\alpha$, IL- 6 , and the expression of proapoptotic protein Bax, confirming that UC increased inflammatory activity and apoptosis in the colon from UC mice model. A previous report pointed out that inflammatory activation in vivo is an important pathological and biological basis for intestinal mucosal injury [12]. In this study, H\&E staining confirmed that UC caused colonic mucosal damage and focal ulcers.

Treatment of AF suppress the apoptosis of colonic mucosal epithelial cells, promote the repair of colon injury, and accelerate the healing of ulcers. Fang et al reported that AF alleviates the progression of UC through the TLR4 signaling pathway [6]. The results from the present study indicate that AF attenuated DSSinduced alterations in apoptosis, inflammatory factors, and ulcer injury. These results suggested that AF might exert a functional role in slowing the progression of UC.
Increasing evidence has suggested that AMPK plays an important role in the inflammatory process and improves intestinal endothelial cell differentiation and barrier function [13]. Moreover, lipopolysaccharide (LPS)-induced acute lung injury is significantly worse in AMPK knockout mice [13]. Macrophage $\beta$-arrestin-1 elevates the expression of inflammatory factors by activating the intracellular AMPK pathway, thereby exacerbating colitis [14]. Hence, it is likely that the occurrence of UC might be related to the AMPK pathway. The results of this study showed that DSS inhibited the activation of AMPK, but treatment with AF re-activated AMPK.

Relatedly, Sun et al reported that AMPK improves the differentiation of intestinal epithelial cell and enhances the function of barrier by regulation of CDX2 expression [15]. This study found that $A F$ increased the expression level of CDX2, indicating that $A F$ caused $U C$ by regulating CDX2. However, AF-mediated regulation of CDX2 is likely not the only way to relieve UC. This study found that the changes in epithelial cell function-related proteins (ZO-1 and villin) caused by DSS are opposite to those of CDX2. However, the expressions of ZO-1 and villin represent a functional improvement of intestinal epithelial cells [13]. Therefore, these results suggest that $A F$ may relieve the symptoms of UC by upregulating CDX2; however, AF did not completely cure UC. The activation of AMPK pathway may be one of the mechanisms responsible for the therapeutic action of AF on UC.

\section{CONCLUSION}

AF attenuates DSS-induced inflammatory damage and apoptosis via AMPK pathway and by regulating CDX2 expression in UC mice, thus indicating that $A F$ is a potential natural therapy for UC. However, it is necessary to further clarify the relationship between inflammatory factors, such as TNF- $\alpha$ and IL-1 $\beta$, and AMPK pathway in order to better understand the pathogenesis and molecular mechanism of UC.

\section{DECLARATIONS}

\section{Conflict of interest}

No conflict of interest is associated with this work.

\section{Contribution of authors}

We declare that this work was done by the authors named in this article and all liabilities 
pertaining to claims relating to the content of this article will be borne by the authors.

\section{Open Access}

This is an Open Access article that uses a funding model which does not charge readers or their institutions for access and distributed under the terms of the Creative Commons Attribution License (http://creativecommons.org/licenses/by/ 4.0) and the Budapest Open Access Initiative (http://www.budapestopenaccessinitiative.org/rea d), which permit unrestricted use, distribution, and reproduction in any medium, provided the original work is properly credited.

\section{REFERENCES}

1. Driessen A: Ulcerative Colitis. In: Pathology of the Gastrointestinal Tract. Edited by Carneiro F, Chaves $P$, Ensari A. Cham: Springer International Publishing; 2017: 669-674.

2. Stefanelli $T$, Malesci A, Repici A, Vetrano S, Danese S. New insights into inflammatory bowel disease pathophysiology: paving the way for novel therapeutic targets. Curr Drug Targets 2008; 9(5): 413-418.

3. Colombel J-F, Sands BE, Rutgeerts P, Sandborn W, Danese S, D'Haens G, Panaccione R, Loftus EV, Sankoh S, Fox I et al. The safety of vedolizumab for ulcerative colitis and Crohn's disease. Gut 2017; 66(5): 839-851.

4. Zhu YL, Wang $L Y$, Wang JX, Wang $C$, Wang $C L$, Zhao $D P$, Wang ZC, Zhang JJ. Protective effects of paeoniflorin and albiflorin on chemotherapy-induced myelosuppression in mice. Chin J Nat Medicines 2016; 14(8): 599-606.

5. Jeong $M-Y$, Park J, Youn $D-H$, Jung $Y$, Kang J, Lim S, Kang M-W, Kim H-L, So H-S, Park R. Albiflorin ameliorates obesity by inducing thermogenic genes via AMPK and PI3KJAKT in vivo and in vitro. Metab 2017; 73:85-99.

6. Fang $X$, Xin $W$, Zhu $X$, Hao $C$, Gang C. Albiflorin attenuates inflammatory injury by regulating the TLR4 signaling pathway and its negative regulating factor
Tollip in experimental models of ulcerative colitis. J Chin Pharm Science 2016; 25:366-372.

7. National Research Council Committee for the Update of the Guide for the CUoL, Animals: The National Academies Collection: Reports funded by National Institutes of Health. In: Guide for the Care and Use of Laboratory Animals. Washington (DC): National Academies Press (US) P Natl Acad Sci USA.; 2011.

8. Wu YN, Xiao YQ, Chen YB. Experimental study on treatment of immune ulcerative colitis in mice by Changlu Enema. Chin J Integr Tradit West Med 2007; 27(1): 65-68.

9. Westerholm-Ormio M, Garioch J, Ketola I, Savilahti E. Inflammatory cytokines in small intestinal mucosa of patients with potential coeliac disease. Clin Exp Immunol 2002; 128(1): 94-101.

10. Guo Z, Ge C, Han B, Qie Z, Kang Y, Zhu Y, Wang W, Tian L, Feng $X$, Ma $X$. Effects of parenteral nutrition of $\omega-3$ polyunsaturated fatty acid, arginine and glutamine on cellular immune status of patients following liver cancer surgery. Trop J Pharm Res 2018; 17(3): 507511.

11. Bhattacharyya S, Dudeja PK, Tobacman JK. ROS, Hsp27, and IKKbeta mediate dextran sodium sulfate (DSS) activation of IkappaBa, NFkappaB, and IL-8. Inflamm Bowel Dis 2009; 15(5): 673-683.

12. Billmeier U, Admyre $C$, Knittel $T$, Zargari A, Neurath MF, Atrey RJG. Tu1706 The TLR-9 Agonist DIMS0150 Leads to the Induction of IL-10 Positive Mucosal Cells in Ulcerative Colitis Patients. Gastroenterology 2014; 146(5): S-822-S-823.

13. Sun $X$, Yang Q, Rogers CJ, Du M, Zhu M-J. AMPK improves gut epithelial differentiation and barrier function via regulating Cdx2 expression. Cell Death Differ 2017; 24(5): 819.

14. Wang $W$, Chen J, Li XG, Xu J. Anti-inflammatory activities of fenoterol through beta-arrestin-2 and inhibition of AMPK and NF-kappaB activation in AICARinduced THP-1 cells. Biomed Pharmacother 2016; 84:185-190.

15. Sun X, Yang Q, Rogers CJ, Du M, Zhu MJ. AMPK improves gut epithelial differentiation and barrier function via regulating Cdx2 expression. Cell Death Differ 2017; 24(5): 819-831. 